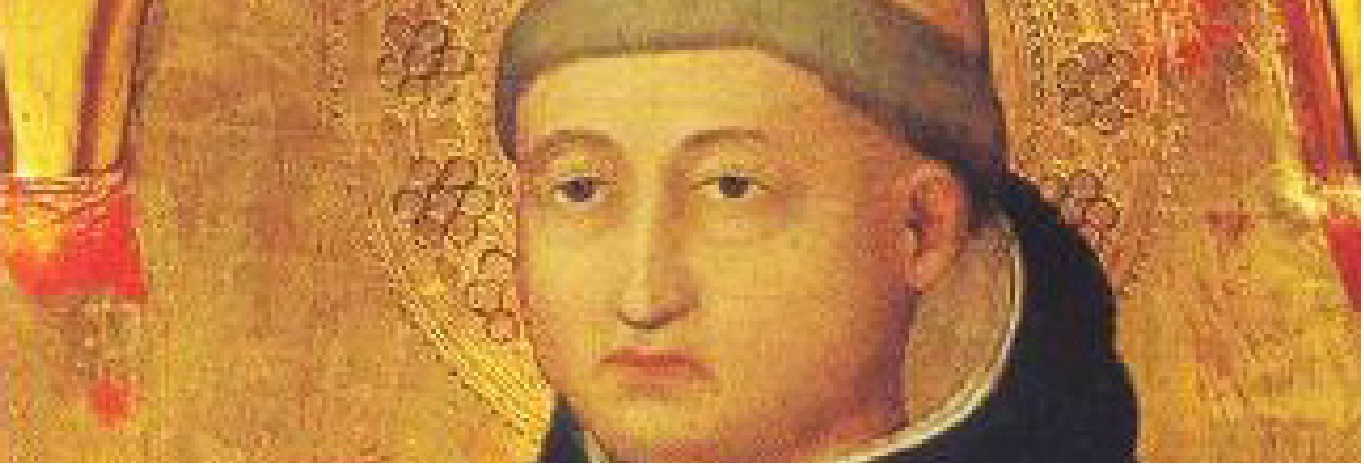

\section{Los primeros principios ontológicos en el pensamiento de Santo Tomás de Aquino}

\author{
The first ontological principles in the thought of Saint Thomas Aquinas
}

\section{Resumen}

Del ser y sus propiedades trascendentales penden los primeros principios ontológicos que son indispensables para cualquier demostración. Sin embargo, la unión entre los primeros conceptos y los primeros principios es tan íntima, que sugiere afinar los detalles de su distinción. De hecho, la relación entre los primeros principios y los primeros conceptos es tal, que los primeros conceptos están contenidos como elementos de los primeros principios. Esa es la razón por la que pensamos que, en este tema, aporta mucho la precisión metafísica de Santo Tomás. Este trabajo está dedicado a presentar la distinción y la primacía de los dos primerísimos principios que, aunque nadie en su sano juicio puede negar, la reflexión filosófica sobre ellos plantea ciertos retos y dificultades que hay que superar. La referencia es, pues, el pensamiento de Santo Tomás que parte del análisis de los presupuestos y fundamentos aristotélicos que constituyen la base para superar cualquier dificultad.

\section{Palabras clave:}

concepto, contradicción, ente, identidad, trascendental.

\begin{abstract}
From the being and its transcendental properties, depend the first ontological principles that are indispensable for any demonstration. However, the union between the first concepts and the first principles is so intimate that refining the details of their distinction is required. In fact, the relationship between the first principles and the first concepts is such that the first concepts are contained as elements of the first principles. This is why we believe that, in this matter, the metaphysical precision of Saint Thomas has much to contribute. This work intends to present the distinction and the primacy of the first two principles since, though no one in their right mind can deny them, philosophical reflection on them poses certain challenges and difficulties that must be overcome. The reference is, then, the thought of St. Thomas starting from the analysis of the Aristotelian presuppositions and foundations that constitute the base to overcome any difficulty.
\end{abstract}

\section{Keywords}

concept, contradiction, entity, identity, transcendental.

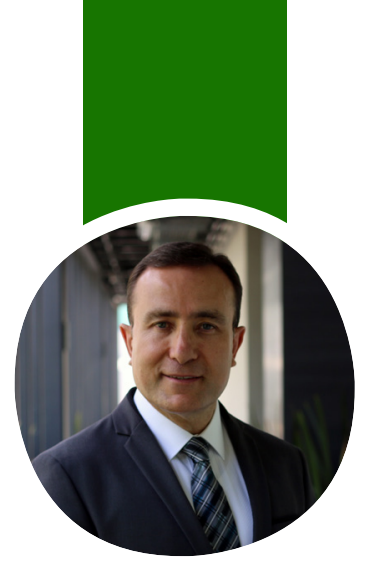

MANUEL OCAMPO PONCE Universidad Panamericana.
Cirujano Dentista (UNAM); Licenciado en Filosofía (Universidad la Salle); Maestro en Humanidades y Doctor en Filosofía (Centro Universitario de la Ciudad de México en colaboración científica con la Fondation de Recherches et D'Editions de Philosophie Neohellenique de Atenas); Doctor en Filosofía y Letras (Universidad Anáhuac del Sur). Profesor investigador en la Universidad Panamericana, Guadalajara, México. Últimas publicaciones: "Aproximación metafísica a la producción de conceptos universales en el pensamiento de Santo Tomás de Aquino", Revista Chilena de Estudios Medievales, Santiago, 2019. "Algunas aportaciones esenciales de Santo Tomás de Aquino a la Filosofía griega clásica", Studium, Madrid, 2019. "Reflexiones metafísicas sobre la ley moral en Santo Tomás de Aquino", Revista Chilena de Estudios Medievales, Santiago, 2019. "Consideraciones metafísicas sobre la posesión de bienes o riquezas en Santo Tomás de Aquino", Studium, Madrid, 2019.

\section{ORCID \#}

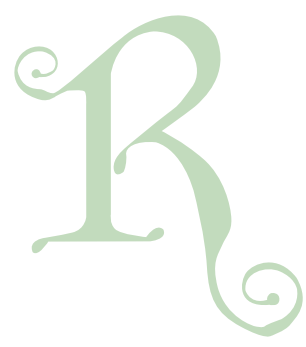

Recepción de artículo: 17-10-2019

Aceptación del artículo: $23-4-2020$ 


\section{NOCIONES PRELIMINARES}

En las Cuestiones disputadas sobre la verdad, Santo Tomás expone la relación de las propiedades trascendentales con los primeros principios afirmando que: 'Debe decirse que, así como en la serie de las proposiciones demostrables es preciso llegar, en último término, a algunos principios inmediatamente evidentes, así también en la investigación de lo que es cada cosa. De lo contrario se procedería hasta el infinito en uno y otro orden, y no podría haber ninguna ciencia ni ningún conocimiento de las cosas'. ${ }^{1}$ Es por eso que la Metafísica hace un gran esfuerzo para llegar a un primer principio que constituye el punto de partida de todos los demás principios. De hecho, Santo Tomás nos alerta al afirmar que no todo principio es un primer principio. ${ }^{2}$ Porque para ser primer principio es necesario que la verdad de la proposición se manifieste a todo sujeto pensante, por el mero análisis del sujeto y del predicado directamente y sin necesidad de demostración. ${ }^{3}$ La duda de estos principios, al igual que toda prueba, supone los principios, de modo que automáticamente confirma su existencia. En lo que se refiere al número de esos principios hay varias propuestas de las que se obtienen cuatro: el de contradicción, el de identidad, el de tercero excluido y el de la razón suficiente. ${ }^{4}$ Sin embargo, nosotros nos centraremos en los dos que, siguiendo a Santo Tomás, consideramos primerísimos y que son el de contradicción y el de identidad destacando la primacía absoluta de uno de ellos.

Respecto de estos primeros principios Aristóteles nos indica, en el libro IV de la Metafísica, que los axiomas se aplican a todos los entes y por eso sostiene que al intelecto, que es capaz de conocer el ente en cuanto ente, corresponde la contemplación de los axiomas. ${ }^{5}$ Dichos principios pertenecen al ente en cuanto ente, de donde se sigue que son principios lógicos, pero, por pertenecer al ente, son también principios ontológicos y, por eso, su estudio corresponde primordialmente a la Metafísica. ${ }^{6}$ Aristóteles también considera importante que el filósofo trate estos principios por su valor universal, que supera las ciencias particulares. ${ }^{7}$
'Precisamente por esto, ninguno de los que especulan parcialmente intenta decir algo acerca de la verdad o falsedad de tales axiomas'. ${ }^{8}$ Pero no cualquier principio puede ser el primero, sino sólo aquel que cumpla con ciertas cualidades. Ese principio debe ser tal que sobre él no pueda quedar ninguna duda. Debe ser el mejor conocido y algo que necesariamente deba conocer cualquiera que pretenda conocer cualquier cosa cuya posesión es previa a todo conocimiento. Ese principio debe ser, intrínseca e inmediatamente evidente, y, por esa razón, el más firme de todos. ${ }^{9}$ Luego sostiene que ese primer principio o axioma es el de contradicción. Porque: 'Es imposible, en efecto, que lo mismo se dé y no se dé simultáneamente en el mismo sujeto y en cuanto ello mismo' ${ }^{10}$ Aristóteles se centra principalmente en su carácter evidente por sí mismo y que, por lo mismo, no necesita demostración. ${ }^{11}$ Sólo puede, en todo caso, refutarse su negación. ${ }^{12}$

Por su parte, Santo Tomás, en el libro I de su Comentario a los Analíticos Posteriores, confirma que toda demostración supone la existencia de principios evidentes e indemostrables que garanticen la certeza de sus conclusiones. ${ }^{13}$ Siguiendo a Aristóteles, explica que del proceso de la experiencia sensible se obtienen los principios de las demostraciones, enfatizando que estos han de ser evidentes e indemostrables. ${ }^{14}$ Pero, además, Santo Tomás también considera algunos argumentos de Boecio, ${ }^{15}$ $y$, por supuesto, de su maestro San Alberto Magno. ${ }^{16} \mathrm{Si}$ suponemos que es posible demostrar algo, entonces es necesario admitir que ciertas proposiciones universales son verdaderas y evidentes. ${ }^{17}$ Santo Tomás atiende a dos aspectos que son el orden lógico y el orden metafísico gnoseológico. Según el punto de vista lógico, Santo Tomás sostiene que son evidentes por sí mismas y no requieren ser demostradas las proposiciones cuyo predicado se encuentra implícito en el sujeto, es decir, aquéllas en las que sólo hay que explicitar el contenido inteligible del sujeto. ${ }^{18}$ Lo único que se requiere, en esos principios, es poseer los términos que conforman la proposición. ${ }^{19} \mathrm{Si}$ conocemos lo que es un todo y una parte, podemos afirmar, con certeza, sin necesidad de demostrar, que 'cualquier todo es mayor que las partes que lo constituyen'. ${ }^{20}$ Santo

1. Tomás de Aquino (S.). De Veritate, q.1, a.1, c.

2. Cfr. Tomás de Aquino (S.). S.Th., I-II, q.6, a.1, ad.1.

3. Cfr. Tomás de Aquino (S.). S.Th., I-II, q.94, a..2. Verit., q.16, a.1.

4. Cfr. Kaufmann Nicolaus Dr. Philos h.c. 1917, p.16.

5. Cfr. Aristóteles. Met., IV, 3, 1005 a 20.

6. "Que es propio del filósofo, es decir, del que contempla la naturaleza de toda substancia, especular también acerca de los principios silogísticos, es evidente... Por consiguiente, es natural que el que más sabe de los entes en cuanto entes pueda enunciar los más firmes principios de todas las cosas. Y éste es el filósofo". Aristóteles. Met., IV, 3, 1005 b 5; Cfr. Aristóteles. Met., III, 2, 997, a 11.15 y Met., IV, 3, 1005 a 33.

7. "Los axiomas son universales en grado máximo y principios de todas las cosas. Y si no corresponde al filósofo, ¿A qué otro corresponderá considerar lo verdadero y lo falso acerca de ellos?" Aristóteles. Met., III, 2, 997 a 12.

8. Aristótles. Met., IV, 3, 1005 a 29

9. Cfr. Aristóteles. Met., IV, 3, 1005 b 11.

10. Aristóteles. Met., IV, 3, 1005 b 19.

11. Cfr. Aristóteles. Met., IV, 4, 1006 a 5.

12. Cfr. Aristóteles. Met., IV, 4, 1006 a 25.

13. Cfr. Valdivia Fuenzalida, José Antonio 2017, pp. 341-361.

14. Cfr. Tomás de Aquino (S.). In II Analíticos Post., lect., 20.

15. Cfr. Tomás de Aquino (S.). In Hebdomadibus, I, Leon. L, p.269.

16. Cfr. Corbini, A. 2006, pp 74-78. Apud. Valdivia Fuenzalida, José Antonio 2017, pp. 341-361.

17. Cfr. Tomás de Aquino (S.). In I Analíticos Post., lect. 7.

18. "... sciendum est quod quelibet propositio cuius predicatum est in ratione subiecti est inmediata et per se nota, quantum est in se" Tomas de Aquino (S.). In I Analíticos Post. Lect. 5

19. Cfr. Tomás de Aquino (S.). In Metaph., IV, lect. 5.

20. Tomás de Aquino (S.). In Analíticos Post., Lect. 5. 
Tomás sostiene que se trata de una verdad per se nota, o conocida por sí misma, a diferencia de otras verdades que son per aliud nota, o conocidas por otras. ${ }^{21} \mathrm{Al}$ respecto, Aristóteles ya había explicado cómo se obtienen los principios evidentes de las demostraciones. ${ }^{22}$ Tanto Aristóteles como Santo Tomás niegan las ideas innatas al afirmar que el conocimiento parte de lo sensible, sin embargo, aclaran que los primeros principios son punto de partida en lo que corresponde al conocimiento intelectual, o, más concretamente al raciocinio que supone el conocimiento sensible. ${ }^{23} \mathrm{~A}$ partir del conocimiento sensible el intelecto elabora conceptos universales simples. ${ }^{24}$

Santo Tomás considera que el intelecto agente hace los inteligibles en acto separando lo universal que se encuentra en los entes singulares. ${ }^{25}$ Para Santo Tomás basta el conocimiento de los términos para formar las proposiciones evidentes. ${ }^{26}$ Tanto Aristóteles como Santo Tomás sostienen que la demostración debe partir de principios que son propios de aquello que se demuestra. ${ }^{27}$ Se trata de esas verdades per se nota que no necesitan ser confirmadas por un silogismo demostrativo en cuanto son evidentes por sí mismas. Basta que el intelecto ponga atención sobre ellas para que adquiera una certeza absoluta. Su predicado forma parte de la definición misma del sujeto; un contenido inteligible que manifiesta por sí mismo su propia verdad, con absoluta certeza, y sin necesidad de otra verdad previamente conocida.

Tanto para Aristóteles como para Santo Tomás, todo aprendizaje se obtiene gracias a la aplicación de los principios per se nota a determinadas materias o a ciertos temas o cosas particulares. ${ }^{28}$ Obviamente estos primeros principios van precedidos por el conocimiento sensible de las realidades sobre las cuales se aplican los principios. De modo que cualquier conocimiento que procede de la experiencia puede, en algún sentido, ser principio. Pero no todo conocimiento obtenido por la experiencia es indemostrable o evidente por sí mismo, sólo el conocimiento per se nota. En efecto, no todo conocimiento que procede de la experiencia sensible puede producir verdades evidentes por sí mismas. Esto sucede porque lo que suscita la formación de una proposición determinada y aquello que permite reconocerla como verdadera, son cosas distintas. No es cuestión del contenido de las verdades sino de aquello por lo cual su contenido es reconocido como verdadero. Como hemos dicho antes, las verdades per se nota, son las que no requieren término medio para ser reconocidas como verdades, porque el predicado pertenece necesariamente al sujeto y eso es captado por el intelecto en cuanto aprehende al sujeto ${ }^{29}$

\section{STATUS QUAESTIONIS}

Una vez establecido de modo muy general lo que es un principio, es preciso indagar cuál es el primer principio. Y aquí veremos que, no sólo para Aristóteles, sino también para Santo Tomás el principio de contradicción tiene gran importancia (una misma cosa no puede ser y no ser al mismo tiempo), mientras que no dan tanta importancia al principio de identidad. De hecho Santo Tomás lo enuncia sólo en su forma lógica. ${ }^{30}$ Además, tanto Aristóteles como Santo Tomás incluyen el principio de tercero excluido que consiste en que entre dos juicios opuestos entre sí contradictoriamente no es posible un tercero. ${ }^{31} \mathrm{Sin}$ embargo, para Santo Tomás, este principio tiene su fundamento en la ley de la contradicción. El fundamento está en que no puede haber un tercero, porque los miembros de la contradicción se relacionan entre sí como el ser y el no ser, como lo verdadero y lo falso, como la afirmación y la negación. De modo que un tercero tendría que ser algo que no sería ser ni no ser, ni verdadero ni falso, ni objeto de afirmación ni objeto de

21. "Verum autem est dupliciter considerabile: uno modo sicut per se notum; alio modo, sicut per aliud notum. Quod autem es per se notum, se habet ut principium; et princiitur statim ab intellectu [...] Verun autem quod est per aliud notum, non statim percipitur ab intellectu, sed per inquisitionem rationis [...]." Tomás de Aquino (S.). S.Th., I-II, q.57, a.2, c. Grosseteste y Alberto Magno ya habían mencionado este tipo de proposiciones que se obtienen del análisis o explicitación de los conceptos. Cfr. Roberto Grosseteste 1981, p.158; Alberto Magno 1890, vol.2, p.5a.

22. Cfr. Aristóteles. Analíticos Posteriores, II, 19.

23. Cfr. Tomás de Aquino (S.). In II Analíticos Post., lect. 20.

24. Cfr. Ibidem.

25. "Posset autem aliquis credere quod solus sensus uel memoria singularium sufficiat ab causandum congnitionem intellibibilem principiorum, sicut posuerunt quídam Antiqui, non discernentes inter sensum et intellectum. Et ideo ad hoc excludendum Philosophus subdit quod simul cum sensu oportet presupoonere talem naturam anime que posit pati hoc, id est que sit susceptiua cognitionis universalis, quod quidem fit per intellectum possibilem, et iterum que possit agere hoc secundum intellectum agentem, qui facit intelligibilia in actu per abstractionem universalium a singularibus." Tomás de Aquino (S.). In II Analíticos Post., lect., 20.

26. "Set tamem experimentum indiget aliqua ratiocinatione circa particularia, per quam confertur unum ad aliud, quod est propium rationis. Puta, cum aliquis recordatur quod talis herba multociens sanauit multos a febre, dicitur esse espertum quod talis sit sanatua febris. Ratio autem non sistit in experimento particularium, set ex multis particularibus in quibus expertus est, accipit aunum commune, quod firmatur in anima, et considerat illud absque consideratione alicuius singularium. Et hoc commune accipit ut principium artis sciencie: puta, quandiu medicus considerauit hanc herbam sanasse Sortem febrientem et Platonem et multos alios singulares hominem, est experimentum; cum autem sua consideratio ad hoc ascendit quod talis species herbe sanat febrientem simpliciter, hoc accipitur ut quedam regula artis medicine". In II Analíticos Post. Lect., 20.

27. Cfr. Aristóteles. Analíticos Post, l, 9, 75b 37-76 a4. Tomás de Aquino (S.). In Analíticos Post., lect. 17, 18, 43.

28. "Processus autem rationis pervenientis ad cognistionem ignoti inveniendo est ut principioa communia per se nota applicet ad determinatas materias et inde procedat in aliquas particulares conclusiones, et ex his in alias [...]". Tomás de Aquino (S.). De Ver., q.11, a.1, c. "Cum autem aliquis huiusmodi universalia principioa applicat ad aliqua particularia, quorum memoriam et experimentum per sensum accipit; per inventionem propriam acquirit scientiam eorum que nesciebat, ex notis ad ignota procedens". Tomás de Aquino (S.). S.Th., I, q.117, c.

29. Vid supra.

30. "quae uni et eidem sunt eadem, sibi invicem sunt eadem". Tomas de Aquino (S.). In IV Met., lect. 3; S.Th., I-II, q.94, a.2.

31. Cfr. Aristóteles. Metafísica, III; Tomás de Aquino (S.). In IV lib. Metaph., lect.4. 
negación. Santo Tomás también nos presenta otro principio que es el de razón suficiente y que consiste en que toda cosa tiene la razón de su ser o bien en sí misma o bien en otra como su causa. ${ }^{32}$ Aunque, si observamos bien este principio, podemos ver que también depende del principio de contradicción en cuanto lo supone necesariamente. Santo Tomás ve claro que, así como las ideas trascendentales exigen la reducción a la idea de ser, analógicamente, los primeros principios inmediatos demostrativos, han de reducirse a un solo principio que es absolutamente primero, y tanto para Aristóteles como para Santo Tomás, ese principio es el principio de contradicción.

El estado de la cuestión radica en que, aun cuando todo parece muy claro, hay quienes han dudado de la primacía del principio de contradicción. Aunque, como hemos visto, para Aristóteles y Santo Tomás la primacía, dentro de los principios, corresponde absolutamente y en todos los aspectos, al principio de contradicción. ${ }^{33} \mathrm{E}$ incluso, posteriormente, con el neotomismo del siglo XIX, varios tomistas, reafirmaron la primacía del principio de contradicción, ${ }^{34}$ en cuanto coincidieron en que el principio de identidad es verdadero, pero no es auténticamente el primero o el axioma por antonomasia, sino que depende del principio de contradicción que tiene la absoluta primacía ontológica. Sin embargo, en el siglo XX, otros filósofos, consideran, en algún momento, la primacía del principio de identidad y desplazan hacia el segundo lugar el principio de contradicción. ${ }^{35}$ Por ejemplo, Garrigou-Lagrange que, a pesar de oponerse a tantas afirmaciones de Suárez, considera, al igual que Suárez, la primacía del principio de identidad al afirmar que es el primer juicio que el intelecto forma. ${ }^{36} \mathrm{El}$ asunto es que, en el orden gnoseológico, Suárez al igual que otros como Zigliara, consideran la primacía del principio de contradicción porque, en el conocimiento, antecede a todos los demás primeros principios. ${ }^{37}$ Sin embargo, como hemos dicho, Garrigou-Lagrange manifiesta que la ley de contradicción se basa en el principio de identidad..$^{38} \mathrm{El}$ argumento es que el ser es ser, y el no ser, no ser, y, por lo tanto, una misma cosa no puede ser y no ser al mismo tiempo y bajo el mismo aspecto. $Y$ en eso, lo que Suárez sostuvo, y tanto Garrigou-Lagranje como Zigliara aceptaron, es que en el orden lógico, todas las pruebas directas se basan en el principio de identidad, mientras que las pruebas indirectas, se basan en el principio de contradicción. ${ }^{39}$ Aunque hay que aclarar que para Garrigou-Lagrange el principio de contradicción viene siendo la fórmula negativa del principio de identidad. ${ }^{40}$ También en el siglo XX, otros como Jacques Maritain siguieron esa tendencia otorgando la primacía al principio de identidad. ${ }^{41}$

Frente a estas posturas, el R.P. Gallus Manser, y otros relevantes tomistas como Eudaldo Forment, Millán Puelles y Abelardo Lobato en el siglo XX, han sostenido que ambos principios son diversos, y que el principio de contradicción posee la primacía absoluta sobre el principio de identidad. ${ }^{42}$ El argumento de Manser que siguen los tomistas mencionados, inicia enfatizando la distinción entre principios partiendo del hecho de que el principio de contradicción no expresa una oposición entre dos proposiciones sino entre dos conceptos que son ser y no ser, en una misma proposición. ${ }^{43}$ Manser parte de la falsedad de la formulación: 'si hay dos juicios contradictoriamente opuestos entre sí uno de los dos tiene que ser falso'. La clave aquí radica en que Manser se está refiriendo a juicios.

Si somos observadores, veremos que Aristóteles ya había dado varias formulaciones entre las que la más sencilla dice así: 'es imposible ser y no ser al mismo tiempo'. ${ }^{44}$ Por su parte, Santo Tomás retoma las fórmulas aristotélicas, sobre todo la que dice: "impossiblile est idem esse et non esse". Lo que Manser señala es que, lo que se opone en esas formulaciones, no son dos juicios sino dos conceptos dentro de la

32. Cfr. Tomás de Aquino (S.). De ente et essentia, c.5.

33. Cfr. Aristóteles. Metafísica, IV, 3, 4, 5, 6; XI, 5 y 6; (en la edición Didot de París corresponde a los libros III y X de la Metafísica). Cfr. Tomás de Aquino (S.). S.Th., I-II, q.94, a.2.

34. Cfr. Gredt, O.S.B. Elementa philosophiae (edit. Tertia), n.550-554; De Maria, S.J. 1897, pp.156-158; Card. Lorenzelli 1896 , pp.241 y ss; Sant Severino 1885, Cap. II, a.4; Liberatore, S.J. 1881, v.1, n. 127-129; Goudin, O.P. 1860, p.IV, disp.I, q.1, a.1; Schneid Sach 1896, p.9; Aoberto Stöckl 1881, t.II, p.6 yss.

35. Entre ellos, se encuentran, por mencionar algunos: Card. Zigliara, O.P., Card. Mercier, Grimmich, O.S.B., Garrigou-Lagrange, O.P., Lehmen-Beck. S.J., Nicolás Kaufmann, Trendelenburg, Gutberlet, Federico Überweg, Willems, Jacques Maritain, Willman, Hugon, O.P. etc. Cfr. DR. P.G.M. Manser, O.P. 1947, p.250.

36. Cfr. Garrigou-Lagrange 1922, pp. 106 y 107; Suárez. Disp. Met., disp. 3, sect., 3, n.4, 7 y 11.

37. Cfr. Suárez. Disp. Met., disp.3, sect., 3, n.9; Zigliara 1874, Summa phil., Log. 55, X (ed. 8). Della luce intellectuale e dell'Ontologismo. Roma, n. 476478.

38. Cfr. Garrigou-Lagrange 1922, 1. c., p. 163, 167 y 170.

39. Cfr. Suárez Disp. Met., 1.c., n.6-9; Garrigou-Lagrange 1922, p.58 y 169; Zigliara. Summ. Phil. Log., 55, VII-X.

40. "le príncipe de non-contradiction n'élant que la formule negative du príncipe d'identité". Garrigou-Lagrange. Le sens., commum, p.107 y 151. "Le príncipe de contradiction apparait comme une formule négative derivée du príncipe affirmative: Ce qui est, est, ce qui n’est pas est ce qui n’est pas, formule courante du príncipe d'identité", Garrigou-Lagrange 1922, p. 163 y 167.

41. "1. Hay para los tomistas una pluralidad de primeros principios; pero también hay entre éstos cierto orden, no en el sentido de que los siguientes al primero de todos pueden ser demostrados partiendo de éste apodícticamente, sino en el sentido de que se puede demostrar -y esto se llama la reducción al imposible- que, si se niega cualquiera de los otros primeros principios de la razón especulativa, se niega necesariamente el primero de todos, es decir, el principio de identidad, y que, si se niega el principio de identidad, no se puede hablar ni pensar, no se puede existir en cuanto ego pensante, en cuanto hombre." Maritain, Jacques 1934, p.1.

42. Cfr. Forment, Eudaldo 1996, pp. 107-124; P.G.M. Manser, O.P. 1947, p.251.

43. Cfr. Ibidem, p.252.

44. Aristóteles. II Met., c.2 y c.4. También formula el principio como: "es imposible que una misma cosa corresponda a lo mismo en el mismo sentido y al mismo tiempo no le corresponda". III Met., c.3. O bien como: "una misma cosa no puede ser simultáneamente afirmada y negada de lo mismo". I Anal. Post., c.11. 
misma proposición: ser y no ser..$^{45}$ Lo que él sostiene es que, lo que hay que considerar en los principios, es la distinción entre el aspecto lógico y el ontológico. Pero para comprender esto, es necesario profundizar en el estudio de lo expuesto por Santo Tomás.

\section{LA DISTINCIÓN DE LOS PRINCIPIOS}

Para aclarar esos puntos en los que se ve una dificultad entre un juicio y un concepto, si consideramos el aspecto lógico, vemos que la formulación del principio, es decir, la proposición, excluye la posibilidad de otra predicación con relación al mismo predicado y sujeto. Se trata de una predicación subjuntiva en la que no se predica nada porque lo que se afirma se excluye mutuamente. El principio de contradicción es el mejor ejemplo de esto en cuanto expresa una oposición inconciliable. En el orden lógico la oposición únicamente puede hacerse por medio de la afirmación y la negación: 'una misma cosa no puede, simultáneamente, ser afirmada y negada de lo mismo' ${ }^{46}$

Sin embargo, no hay que perder de vista que la oposición lógica tiene su fundamento en la realidad. Y aquí el trascendental aliquid se puede considerar como el ente en cuanto que es opuesto contradictoriamente con el no-ente, fundándolo en la unidad trascendental, puesto que no se puede añadir al ente la negación de la identidad con los demás, si éste no tiene la negación de la división interna. El trascendental "algo" añade al ente la división externa, pero ésta no se sigue inmediatamente de la noción de entidad como sucede en otros trascendentales, sino que procede del ente por medio de la unidad. ${ }^{47}$ En estos casos lo que se manifiesta, en última instancia, es que lógicamente se expresa la incompatibilidad absoluta del ser y del no ser. ${ }^{48} \mathrm{El}$ ser del ente es algo real que el intelecto conoce con anterioridad a cualquier otro conocimiento y que excluye el no ser de manera inmediata. Esa oposición entre ser y no ser es lo que expresa el principio de contradicción. Se trata de una proposición que expresa una oposición real entre el ser y el no ser. Todas las diferencias de las cosas se reducen a la oposición trascendental entre el ser y el no ser. Por eso, podemos considerar que el principio de contradicción es anterior al de identidad, porque en la relación esencial del ser y del no ser está la razón de su oposición y de su irreductibilidad.

En cuanto al principio de identidad, este se enuncia como: 'cada ser es lo que es, es idéntico a sí mismo y, por tanto, un mismo ser, un uno' ${ }^{49}$ Sin embargo, como hemos visto antes, Aristóteles y Santo Tomás no lo manifiestan como un axioma ontológico. De hecho, Santo Tomás manifiesta un claro conocimiento sobre el principio del que depende.50 En lo que se refiere a Aristóteles, él lo enuncia diciendo: 'Todo lo que es verdadero tiene que convenir absolutamente consigo mismo', ${ }^{51}$ o como: 'una misma cosa tiene que ser lo mismo que ella misma' ${ }^{52}$ De esto se deduce su radical coincidencia con la unidad del ser. ${ }^{53}$ Lo cual se manifiesta en la relación esencial o la oposición entre el ser trascendental y el no ser. Por eso se ha llegado a pensar que cualquier intento por formular el principio de identidad es tautológico, porque lo que es, es, y lo que no es, no es. Y, por eso, en cualquier intento de formulación el predicado expresa lo mismo que el sujeto. Sin embargo, aquí hay que añadir que la tautología se supera cuando distinguimos el orden lógico del ontológico porque, en este caso, como en el caso de las propiedades trascendentales del ser, el predicado añade algo lógicamente distinto del sujeto. Aunque entre el sujeto y lo que se predica de él no haya distinción real, existe una distinción de razón que evita la tautología. Lo que la unidad añade al ser es una distinción de razón en cuanto nuestro intelecto es discursivo y para conocer divide incluso lo absolutamente simple conociendo primero el ser, luego el no ser y luego la unidad indivisible que es lo mismo que el ser. ${ }^{54}$ Por eso, en el orden lógico o en el orden del conocimiento humano existe una diferencia entre el ser y la unidad, según como nosotros aprehendemos. ${ }^{55}$ Sin embargo, más allá de eso existe una distinción ontológica entre los principios de identidad y de contradicción, porque el principio de identidad expresa la identidad o la unidad real del ser trascendental consigo mismo en la que se fundamentan potencialmente la unidad y la independencia de todas las cosas existentes en el universo. Cada cosa posee un ser propio que las constituye esencialmente. El principio de contradicción expresa la oposición real entre el ser y el no ser en la que se fundamentan potencialmente la multiplicidad y la diversidad reales de todas las cosas que existen en el universo. La oposición entre el ser real y el no ser fundamenta absolutamente la distinción real entre las cosas. Como vemos, cada principio expresa una cosa distinta objetiva y realmente y, por eso, los dos principios son objetivamente distintos.

De aquí que Manser haya señalado que no comprende cómo algunos autores tomistas, y otros autores idealistas modernos como Hegel, hayan sostenido que el principio de contradicción no es más que la fórmula negativa del principio de identidad. ${ }^{56}$ De hecho, Manser sostiene que la fórmula negativa del principio de identidad sería: el ser es no ser y el no ser es ser. De ese modo queda claro que los que cometen ese error sostienen que el principio de contradicción es la fórmula negativa derivada del principio de identidad. Para ellos el ser no puede ser al

45. Cfr. Dr. P.G.M. Manser, O.P. 1947, p.252.

46. Cfr. Ibidem, p.253

47. Cfr. Forment, Eudaldo 1996, p.118.

48. Cfr. Aristóteles., De interpret., c.6.

49. "Unumquodque est indivisibile ad seipsum"; "est unum sibi ipsi". Tomás de Aquino (S.). In. Met., lect. 15; "verum est esse quod est". Tomás de Aquino (S.). In IV Met., lect. 4.

50. "Quae uni et eidem sunt eadem, sibi invicem sunt eadem". Tomás de Aquino (S.). In IIV Met., lect. 3; S.Th., I-II, q.94, a2.

51. Aristóteles. In An. Post., c.32.

52. Aristóteles. Metafísica, IV, c.9.

53. Cfr. Ibidem.

54. "Non est nugatio cum dicitur ens unum, quia unum addit aliquid secundum rationem supra ens". Tomas de Aquino (S.). S.Th., I, q.11, a.1, ad. 3; Cfr. Tomás de Aquino (S.). S.Th., q.11, a.2, ad.4.

55. Tomás de Aquino (S.). S.Th., I, q.11, a.2, ad.4.

56. Überweg, System der Logik, 77, p.252. Apud. Dr. P.G.M. Manser, O.P. 1947, p.256. En una línea parecida se encuentran Suárez, Leibniz y Wolff. 
mismo tiempo no ser. ${ }^{57}$ Lo que Manser señala es que ‘ ide la oposición entre el ser y el no ser, que se expresa en el principio de contradicción, se deduce la validez del principio de identidad, y no a la inversa!'. ${ }^{58}$ Lo primero es el ser del ente, lo segundo es la oposición del ser con la nada y, posteriormente la oposición del ente a todo lo demás.

\section{PRIMACÍA DEL PRINCIPIO DE CONTRADICCIÓN}

Es un hecho que el ser no puede, al mismo tiempo, ser y no ser. En eso radica la primacía ontológica de un principio y, por tanto, el principio de contradicción cumple con las exigencias de Aristóteles y Santo Tomás, ya que no hay nada anterior de modo que, el principio de contradicción, supone todos los otros principios..$^{59}$ De hecho, Santo Tomás, también en la Suma Teológica, manifiesta claramente la primacía del principio de contradicción. ${ }^{60}$ Santo Tomás aclara muy bien eso en su comentario a Aristóteles. ${ }^{61}$ La razón última de la identidad, es decir, de que el ser sea y el no ser, no sea, es que de no ser así, sería y no sería al mismo tiempo, lo cual es negado por el principio de contradicción. El ser y el no ser no pueden identificarse jamás porque una misma cosa no puede pensarse como ente y no ente al mismo tiempo y eso es justo lo que pone de manifiesto el principio de contradicción. Se trata de la relación trascendental entre el ser y el no ser la cual constituye la explicación o la razón ontológica de la identidad del ser que expresa el principio de identidad. Por eso el principio de contradicción es ontológicamente anterior y fundante de todo otro principio incluido el de identidad. ${ }^{62}$

Hemos visto que algunos pensadores consideran que la proposición "ser es ser" es anterior a la proposición "ser y no ser no puede ser simultáneamente". Sin embargo, también hemos visto la primacía ontológica que otorgan Aristóteles y Santo Tomás, junto con otros autores, al principio de contradicción. El ente es lo primero que la inteligencia percibe, mientras el principio de contradicción es el primer juicio de la segunda operación del intelecto. ${ }^{63}$ Ser y no ser, son los primeros conceptos que la inteligencia nos presenta y de los cuales el intelecto formula el primer juicio que consiste en que el ser no puede ser simultáneamente no ser. ${ }^{64}$ Por eso, para Santo Tomás queda claro que todo lo que el intelecto humano conoce, supone el principio de contradicción que no es sino el primer juicio que el intelecto elabora de manera espontánea. ${ }^{65}$

En lo que se refiere al principio de identidad, el ser es idéntico a sí mismo y el conocimiento de la unidad del ser equivale al conocimiento de la indivisibilidad del ser. Lo uno es el ser en cuanto indivisible. En el principio de identidad hay que ser conscientes de que al ser trascendental se opone el no ser. De modo que supone una distinción entre ser y no ser. ${ }^{66} \mathrm{El}$ conocimiento de la indivisibilidad del ser supone que tenemos las ideas de ser y no ser, así como la distinción entre ambas. ${ }^{67}$ Pero si no conociéramos que el ser y el no ser no pueden ser simultáneamente lo mismo, porque no se distinguirían, no podríamos entender el principio de identidad. Por eso, el conocimiento de la indivisiblidad del ente, es decir, el de la identidad del ser, supone el conocimiento del principio de contradicción. Porque, temporalmente, el primer concepto que se produce es el de ser, luego el de no ser y el primer juicio es el de contradicción en el que se manifiesta como irreductible la distinción entre estos dos conceptos. De donde se sigue la indivisibilidad que es la unidad o la identidad del ser. ${ }^{68}$ De modo que, el hecho de que sepamos que el ser es idéntico a sí mismo, uno e indivisible, supone que conocemos que ser idéntico y no ser idéntico, ser uno y no ser uno, ser indivisible y ser divisible, no son lo mismo. ${ }^{69} \mathrm{El}$ intelecto humano es discursivo, conoce mediante la división, la comparación; separando y uniendo lo que conoce en base a oposiciones, pasando de la potencia al acto. ${ }^{70}$

Hasta ahora, podemos vislumbrar que el principio de contradicción no sólo tiene primacía ontológica, sino también lógica, al ser considerado como el primer principio de toda demostración. Desde el inicio de este trabajo vimos que Aristóteles y Santo Tomás sostienen que los primeros principios no demuestran nada precisamente porque son primeros, comunes y necesarios para toda demostración. ${ }^{71}$ No hay que demostrarlos ni demuestran porque son inmediatamente e intrínsecamente evidentes. ${ }^{72}$ En Aristóteles y Santo Tomás, todas las

57. Cfr. Garrigou-Lagrange 1922, p.163.

58. Manser 1947, p.257.

59. Cfr. Aristóteles. Metafísica III, c.3; Tomás de Aquino (S.). In IV Met., Lect. 2; In XI Met., Lect.5.

60. "Illud quod primo cadit in apprehensione est ens, cuius intellectus includitur in ómnibus quaecumque quis apprehendit. Et ideo primum principium indemostrabile est, quod non est simul affirmare et negare, quod fundatur supra rationem entis et non entis et super hoc principio omnia alia fundantur". Tomás de Aquino (S.). S.Th., I-II, q.94, a.2.

61. Cfr. Tomás de Aquino (S.). In IV Met., lect. 3; In XI Met., lect. 5 y 6.

62. "quod fundatur supra rationem entis et non entis et super hoc principio omnia alia fundantur". Tomás de Aquino (S.). S.Th., I-II, q.94, a.2.

63. "ideo hoc etieam principium (impossibile est esse et non esse simul) est naturaliter primum in secunda operatione intellectus sc. componentes et dividentis". Tomás de Aquino (S.). In IV Met., lect. 2.

64. Cfr. Tomás de Aquino (S.). In XI Met., lect.5.

65. Cfr. Aristóteles. III Met., c.3. "Nec aliquis potest secundum hanc operationem (intellectus dividentis et componentis) aliquid intelligere nisi hoc principio intelecto." Tomás de Aquino (S.). In IV Met., lect.2.

66. "Unum vero, quod convertitur cum ente, non addit supra ens nisi negationem divisiones". Tomás de Aquino (S.). Pot., q.7.

67. Cfr. Tomás de Aquino (S.). S.Th., I, q.11, a.2, ad.4.

68. "Primum, enim, quod in intellectum cadi test ens; secundum, vero, negatio entis; ex his autem duobus sequitur tertio intellectus divisiones; quarto autem sequitur in intellectu ratio unius". Tomas de Aquino (S.). Pot., q.7, ad.15; S.Th., I, q.11, a.2, ad.4.

69. Cfr. Dr. P.G.M. Manser, O.P. 1947, p.262.

70. Cfr. Tomás de Aquino (S.). S.Th., I, q.85, a.5.

71. Cfr. Aristóteles. Analíticos Post., c.32; Tomás de Aquino (S.). In I Analíticos Post., lect. 41.

72. "ens et non ens [...] non contingit ídem essse et non esse, quae uni et eidem sunt aequalia, sibi invencem sunt aequalia". Tomás de Aquino (S.). In I Analíticos Post., lect.41. 
demostraciones tanto directas como indirectas suponen el principio de identidad, pero en ambas demostraciones, el principio de identidad, supone el principio de contradicción. Porque, como lo hemos visto, sin el principio de contradicción no se puede dar la identidad. ${ }^{73}$ Con esto se ve el lugar que Santo Tomás reconoce al principio de contradicción. ${ }^{74}$ En lo que se refiere a Aristóteles, coloca al principio de contradicción como el más seguro de todos los principios en cuanto deja fuera la posibilidad de tener errores. ${ }^{75}$ Y Santo Tomás lo refuerza al decir que nadie puede equivocarse acerca de él, porque es conocido por todos. $Y$ es que, al ser primero, no supone nada antes y es supuesto para todo conocimiento de modo que no necesita ser probado sino que se conoce directa y espontáneamente, porque se abstraen sus términos que son ser y no ser. ${ }^{76}$ Es evidente por sí mismo, de modo que tanto Aristóteles como Santo Tomás sostienen que pretender probarlo es un absurdo que conduce a cerrar las posibilidades de todo conocimiento objetivo de las cosas. ${ }^{77}$

\section{CONCLUSIÓN}

Como lo hemos visto antes, Santo Tomás sostiene que: 'aquello que primeramente concibe el intelecto, como lo más evidente, y en lo cual vienen a resolverse todas las demás concepciones, es el ente, como dice Avicena al comienzo de su Metafísica (Tract. I, cap.6). ${ }^{178} \mathrm{El}$ primer concepto que el intelecto produce es el ente: 'Lo primero que cae en la concepción de nuestro intelecto es el ente [...] el ente es el objeto propio del intelecto, y así es el primer inteligible, así como el sonido es lo primero que se oye' ${ }^{79}$ Nada puede añadirse al ente que no sea un modo del mismo ente, que se encuentre en él, de modo que, en el caso de su concepto, es necesario explicitar lo que se encuentra implícito en el mismo concepto de ente..$^{80}$

En lo que se refiere a las propiedades trascendentales, Santo Tomás se refiere a ellas como 'una clase de modo de ser que acompaña de forma general a todo ente'. ${ }^{81}$ Lo que los trascendentales añaden al ente no es nada real, puesto que cada uno de ellos tiene el mismo contenido que el del ente. Lo que añaden al explicitarlo es algo meramente de razón. No es algo real, sino conceptual. ${ }^{82} \mathrm{El}$ concepto de unidad se genera a partir del principio de contradicción, primer principio del pensamiento y ley general de todo ente porque, como lo había dicho Aristóteles, es 'imposible que un mismo atributo se dé y no se dé simultáneamente en el mismo sujeto y en un mismo sentido' ${ }^{83}$ Y cuando afirma esto se refiere no solo al orden de los conceptos, sino también al orden de la realidad. Por eso Santo Tomás lo explica diciendo que 'lo primero que cae en el entendimiento es el ente; lo segundo es la negación del ente; de estos dos se sigue lo tercero: el concepto de división (de que algo es entendido como ente, y se entiende lo que no es el ente, se sigue, en el entendimiento que está dividido de ello); en cuarto lugar, se sigue, en el entendimiento, la razón de uno, es decir, en cuanto se entiende que el ente no está dividido en sí mismo' ${ }^{84}$ El concepto de división aparece con la oposición contradictoria entre el ente y el noente, como está establecido en el principio de contradicción. En cuanto al principio de identidad, este se funda sobre la unidad del ente, de modo que es posterior al principio de contradicción.

De modo que ahora podemos concluir con Aristóteles y Santo Tomás que el principio de contradicción es primero porque todos los demás principios, incluyendo el de identidad, se validan en el principio de contradicción. Y es que al principio de contradicción corresponde la última razón de la unidad del ser. Pero, además es el primer juicio que el intelecto humano realiza puesto que, aun el principio de identidad, supone la incompatibilidad del ser con el no ser. Por eso es tan originario, porque no se puede prescindir de él, en cuanto supone todas las demostraciones y es anterior a todos los principios demostrativos; negarlo conlleva la imposibilidad de la negación misma, es decir, no se puede negar sin afirmar su primacía.

El principio de contradicción expresa los dos términos que lo constituyen, a saber, el del ser y el del no ser trascendentales y esto tanto en el plano ontológico como en el lógico. Lo primero conocido es el ente, aunque de modo inconsciente y anterior a todo otro tipo de conocimiento. No hay conocimiento discursivo si no se aprehende el ente, de modo que la reflexión científica sobre el ente lo supone como concepto inicial, el ente y la exclusión del no ente. Lo mismo sucede con el principio de contradicción, es el primer juicio inconsciente, natural y espontáneo que luego se hace consciente mediante la reflexión sobre este primer juicio originario, lo que hace que su reflexión sea más oscura y pueda dar lugar a confusión por ser científica y, por tanto, discursiva. De hecho, Santo Tomás sostiene que el principio de contradicción, como primer juicio originario, es realizado por el intelecto humano aun sin saber que juzga. ${ }^{85} \mathrm{El}$ intelecto supone ese juicio en total inconsciencia de que es la primera vez que juzga, y que ese juicio que es el principio de contradicción es un juicio real que es inmediatamente evidente e

73. Cfr. Aristóteles. II Anal. Prior., c.2; Aristóteles. I Anal. Post., c.32; Tomás de Aquino (S.). In IV Met., lect. 2.

74. "Et propter hoc omnes demostrationes reducunt suas propositiones in hanc propositionem sicut in ultimam opinionem ómnibus communem". Tomás de Aquino (S.). In IV Met., lect.2.

75. Aristóteles. Metafísica III, c.3 y 4; Metafísica X, c.5, $(589,1)$.

76. "Manifestum est ergo quod certissimum principium sive firmissimum tale debet esse, ut circa id non possit errari et quod non sit suppositum et quod adveniat naturaliter." Tomás de Aquino (S.). In. IV Met., lect.2.

77. Cfr. Tomás de Aquino (S.). In IV Met., lect. 2; Aristóteles. Met., III, c.4 (504, 11 y ss) y Met., X, c.5 (589, 5 y ss). También véase: Aristóteles. Met., III, c.6 y Tomás de Aquino (S.). In IV Met., lect. 3, 4 y 5 y X Met., c.5 y 6.

78. Ibidem.

79. Tomás de Aquino (S.). S.Th., 1, q.5, a.2, c.

80. Forment, Eudaldo 1996, p. 110.

81. Tomás de Aquino (S.). De Veritate, q.1, a.1, c.

82. Cfr. Forment, Eudaldo 1996, p.111.

83. Aristóteles. Metafísica. IV, 3, 1005b, 19-20.

84. Tomás de Aquino (S.). De Potentia, q.9, a.7, ad. 15. Apud. Forment, Eudaldo 1996, p.115.

85. Cfr. Tomás de Aquino (S.). In IV Met., lect. 2. 
intrínsecamente evidente en sí. El intelecto lo formula sin saber tampoco, por qué ese juicio es inmediatamente e intrínsecamente evidente. Eso lo realiza cuando se hace consciente y estudia científicamente, y más propiamente, metafísicamente, dicho principio. Como quedó asentado en este trabajo, no es posible negar el principio de contradicción sin suponerlo en la misma negación. Porque el principio de contradicción responde a la misma naturaleza de nuestro intelecto que procede naturalmente y necesariamente dividiendo y componiendo, lo cual supone siempre el ser abstraído y su negación. Su primer juicio supone y manifiesta la incompatibilidad objetiva y ontológica del ser y el no ser como términos fundamentales del juicio. Eso lo realiza naturalmente y espontáneamente. Esa es la razón por la que Santo Tomás defiende su primacía. ${ }^{86}$ Además, el principio de contradicción es tan amplio como el ser. Es válido en todo el orden real, porque el ser trascendental, que contiene potencialmente todos los conceptos reales, es real. Por eso, hay que volver a resaltar, que el ser lógico o ente de razón, es una representación que la mente elabora a partir del ser real, lo cual ubica al principio de contradicción como fundamental tanto en el plano lógico como en el ontológico de la realidad que abarca y trasciende lo puramente mental.

Además de todo lo anterior podemos obtener un corolario al deducir que tal y como el ser es análogo con analogía de proporción o atribución intrínseca y de proporcionalidad propia, también el principio de contradicción es análogo con esos tipos de analogía. El principio de contradicción expresa la oposición irreductible del ser trascendental y del no ser, y, por tanto, en el principio de contradicción está virtualmente contenida toda la multiplicidad y la distinción abstracta, tanto en el plano lógico como en el ontológico. Es en esta multiplicidad y distinción abstracta que tiene su razón más profunda. De aquí que haya tanta diversidad en la aplicación del principio de contradicción a todos los objetos a los que se aplica. Se trata de un principio que es análogo como lo es el ser. Y, por lo mismo, los fundamentos de este principio tienen sus raíces en la doctrina del acto y la potencia que explican, a su vez, la analogía puesto que, bajo estos supuestos, virtualmente o potencialmente una cosa puede ser muchas opuestas, si se considera desde distintos puntos de vista, pero actualmente no puede serlo, si se considera desde el mismo punto de vista. ${ }^{87}$

86. "adveniat quasi per naturam habenti ipsum, quasi ut naturaliter cognoscatur". Tomás de Aquino (S.). In IV Met., lect. 2.

87. Cfr. Aristóteles. Metafísica III, c.5 (209,3); Cfr. Tomás de Aquino. In IV Met., lect. 3. 
- Alberto Magno. Super librum Posteriorum Analyticorum primum, Tract. I, c 2, París, Ed. Borgnet Luis Vives, vol.2, 1980.

- Aoberto Stöckl. 1881. Lehrbuch der Philosophie, t.Il, from: https://www.worldcat.org/title/lehrbuch-der-philosophie/oclc/27847856

- Aristóteles. Physics (I-II). Trad. P.H. Wicksteed y F. M. Cornford, Harvard-Heinemann, Cambridge-London, Loeb Classical Library, 1980.

- Metafísica, Segunda edición trilingüe. Madrid, Gredos, 1982.

- - Posterior Analytics, Topica, Cambridge-London. Trad. H. Tedennick y E.S. Forster, Harvard-Heinemann, Loeb Classical Library, 1989.

- - Obras completas. Trad. Carlos Megino Rodríguez, Tomás Calvo y Miguel Candel Sann Martí. Madrid, Gredos, 2011.

- Canals Vidal, F. El "lumen intellectus agentis" en la ontología del conocimiento de santo Tomás, en Convivium I, 1956. Reproducido en 1981 Cuestiones de fundamentación. Barcelona: Bublicacions i Edicions de la Universitat de Barcelona, 11-40, 1981.

- - Sobre la esencia del conocimiento. Barcelona: PPU, 1987.

- Card. Lorenzelli, B. Philosophiae theoreticae institutiones secundum doctrinam Aristotelis et S. Thomae Aquinatis:quas in Pontificio Collegio de Propaganda Fide. Romae P. Cuggiani, 1890.

- Carlos Arthur R. do Nascimento. "Nota sobre a questao dos universais em Tomás de Aquino." Revista Española de Filosofía medieval, n. 7, pp. 137-142, (2000).

- Contreras, Sebastián. "La inteligibilidad de la naturaleza y su vinculación con el conocimiento de los universales." Anuario de Estudios medievales. No. 41, 1, pp. 375-378, (2011).

- Corbini. A. La teoría de la sciencza nel XIII secolo. I commenti agli Analitici Secondi. Firenze, SISMEL-Edizioni del Galluzo, 2006.

- De Maria, Michele, S.J. 1897, Compendium Logicae et Metaphysicae. Roma. Romae: Ex oficina typographica Forzani et socii (IS), Forzani \& C., 1897.

- Derisi, Octavio Nicolás. La doctrina de la Inteligencia de Aristóteles a Santo Tomás, Buenos Aires, Cursos de cultura católica, 1945.

- Estudios de Metafísica y Gnoseología. II Gnoseología. Buenos Aires, E.D.U.C.A., 1985.

- Dr. P.G.M. Manser, O.P. La esenia del tomismo. Trad. De la 2da edición alemana por Valentín García Yerba, Madrid, Consejo Superior de Investigaciones Científicas. Insituto "Luis Vives" de Filosofía, 1947.

- Forment, Eudaldo. Lecciones de Metafísica. Madrid. Ediciones Rialp, 1992.

- - "Autoconciencia y ser en Santo Tomás". Revista Española de Filosofía Medieval 8 (2001).

- - "La sistematización de Santo Tomás de los trascendentales". Contrastes. Revista interdisciplinar de Filosofía, vol. I, pp. 107-124. (1996).

- García López, Jesús. La abstracción según Santo Tomás, from: http://dadun.unav.edu/bitstream/10171/37680/1/201802\%20CAF\%20 250\%20\%282013\%29.pdf ,1971.

- - "La abstracción según santo Tomás". Anuario Filosófico, Vol. 8, No.1, pp. 205-221, (1975).

- Metafísica tomista. Ontología, Gnoseología y Teología natural. Pamplona. EUNSA, 2001.

- Garrigou-Lagrange. Le sens commum, la philosophie de l’etre et les formules dogmatiques, París, 3me éd., 1922.

- Goudin, O.P. Philosophia juxta inconcussa tulissimaque d. Thomae dogmata. (1860), from: http://bdh-rd.bne.es/viewer.vm?id=0000164324\&page=1, 1784.

- Gredt, Joseph. O.S.B. Elementa philosophiae Aristotelico-Thomisticae. Vol.1 (edit. Tertia), Freiburg, Herder, 1926.

- Heinrich M Shmidinger. Filosofía cristiana en el pensamiento católico de los siglos XIX y XX, Tomo 2, vuelta a la herencia escolástica. Editores: Emerich Coreth SJ, Walter M Neidl, Pfligersdorffer, Trad. Eloy Rodríguez Navarro. Madrid, Encuentro Ediciones, 1994. 
- Kaufmann Nicolaus Dr. Philos h.c. Elemente der Aristotelischen ontologie: mit berücksichtigung der Weiterbildung durch den hl. Thomas von Aquin und neuere Aristoteliker: Leitfaden für den unterricht in der allegemeinen metaphysik. Luzern, Druck und Verlag von Raber \& Cíe, 1917.

- Kaufmann Nicolaus., Dr. Mansion Auguste, Dr. "Elemente dere aristotelischen Ontologie, mit Berücksichtigung der Weiterbildung durch den hl. Thomas von Aquin und neuere Aristoteliker. Leitfaden für den Unterricht in der allegemeinen Metaphysik. Zweite verbesserte Aufalage". In: Revue neó-scolastique de philosophie. 22e année, n.87, pp.335-336, (1920).

- Liberatore, S.J. Institutiones Philosophiae, v.1. Roma, nov. editio. 3 vol. in $8^{\circ}$ Prati. Net., 1881.

- Maritain, Jacques. Los primeros principios de la razón especulativa. Transcripción parcial de las lecciones $5^{a}$, $6^{\mathrm{a}}$ y $7^{\mathrm{a}}$ del libro Siete lecciones sobre el Ser y los primeros principios de la razón especulativa, de 1934, from: http://www.jacquesmaritain.com/pdf/04_MET/08_M_PrimPrinc.pdf

- Millan Puelles, Antonio. Fundamentos de Filosofía. Undécima edición. Madrid, RIALP, S. A., 1981.

- Roberto Grosseteste. Commentarius in posteriorum analyticorum libros I, c,8, ed. Rossi, Olschki Editore, Firenze, 1981.

- Sant Severino. Institutiones seu elementa philosophiae christianae. Ontología, 1885.

- Schneid Sach. Grundzüge der Metaphysik. Paderborn, Schöningh, 1896.

- Suárez, Francisco. Disputaciones. Metafísicas. Edited by S. Rábade, Madrid, Gredos, 1961.

- Sellés, Juan Fernando. El hábito conceptual y la distinción entre los universales lógicos y reales según Santo Tomás de Aquino. México. Dianoia vol.53, n.61, (2008).

- Tomás de Aquino (S.). Summa Contra Gentiles, Turín-Roma, ed. Marietti, $11^{\circ}$ reimpresión, 1905.

- - Quaestiones Quodlibetales, 9 edición a cargo de R. Spiazzi.Turín-Roma, ed. Marietti, 1956.

- - Summa Theologiae. Primae Pars, vol. I, Madrid, Biblioteca de Autores Cristianos, 1961.

- - Summa Theologiae. Prima Secundae, vol. II, Madrid, Biblioteca de Autores Cristianos, 1962.

- - Summa Theologiae, Secunda Secundae, vol. III, Madrid, Biblioteca de Autores Cristianos, 1963.

- - Summa Theologiae. Tertia Pars, vol. IV, Madrid, Biblioteca de Autores Cristianos, 1964.

- - B. R. Opera Omnia, ut sunt in Indice thomistico, additis 61 acriptis ex aliis medii aevi auctoribus, curante Roberto Busa, Sj (Frommann Verlag Günter Holz-book KG, Stutgart-Bad Constatt, 1980.

- - Opúsculos y questiones selectas. Tomo I (Filosofia 1). Madrid, Bibiloteca de Autores Cristianos, A.C., 2001.

- - Opúscuos y questiones selectas. Tomo III (Teología 1). Madrid, Biblioteca de Autores Cristianos A.C., 2005.

- - Summa Contra los Gentiles, ed. Bilingüe, con texto de la ed. Leonina, dirigida por L. Robles Carcedo y A. Robles Sierra, introducciones por Eudaldo Forment Giralt, 2 vols. Madrid. Biblioteca de Autores Cristianos, 2007.

- - Cuestiones disputadas sobre la verdad Tomos I y II, Turín-Roma, Pamplona, EUNSA, 2016.

- - Alarcón, E, S. Thomae de Aquino Opera omnia (Corpus Thomisticum, Pamplona 2000s.: http//www.anav.es/filosofía/ALARCON/.

- Tomasz Duma. "The role of Existential Judgements in Knowing the Esistence of Beings". Espíritu LXIII n. 148, 317-331, (2014).

- Überweg, Friederich. System der Logik. Bonn. Bei Adolph Marcus, 1874.

- Valdivia Fuenzalida, José Antonio. "El origen de los primeros principios según Tomás de Aquino. Una dificultad interpretativa". Anales del Seminario de Historia de la Filosofía 34 (2) pp. 341-361, (2017).

- Zigliara. Summa phil., Log. 55, X (ed. 8). "Della luce intellectuale e dell’Ontologismo". Roma, n. 476-478, (1874). 